\title{
Multiple description video coding for underlay cognitive radio network
}

\begin{abstract}
Cognitive radio (CR) with spectrum sharing allows new or secondary devices to co-exist with primary (licensed) users (PU) in accessing the spectrum. This is known as underlay CR. It allows the secondary users (SU) to transmit multimedia data services (video transmission) at low power and low data rate when the PU is using the spectrum. Hence SU can still enjoy uninterrupted video services with minimum tolerable quality. However, problem arises when SUs are subjected to interferences mainly from PU and other SUs. The objective of this paper is to provide error-free video transmission to $\mathrm{SU}$ in the underlay CR transmission by using an error resilience method, namely Multiple Description Coding (MDC). Since the underlay mode CR is characterized by low power, low data rate and possibly high packet loss rate, base layer video streaming of a Scalable Video Coding (SVC) with MDC is a feasible solution. The base layer video is coded using MDC with even and odd frames generating two descriptions. Simulation results show that transmitting video in the underlay CR using MDC perform better objectively and subjectively than using a single description coding (SDC).
\end{abstract}

Keyword: Cognitive radio; Underlay; Multiple description video coding; Scalable video coding; Error resilience 